\title{
EFFECT OF AGE, GENDER AND DURATION OF DIABETES MELLITUS ON THYROID DYSFUNCTION IN PATIENTS OF DIABETIC
}

\author{
Kamran Ali, Sajid Ali Shah*, Syed Murtaza Ali**, Raja Jibran Akbar**, Syed Qasim Raza****, Sadia Kadir**** \\ 66 Medical Battalion, Lahore Pakistan, Combined Military Hospital Skardu/National University of Medical Sciences (NUMS) Pakistan, **Combined Military \\ Hospital Loralae/National University of Medical Sciences (NUMS) Pakistan, ${ }^{* * *} 123$ Medical Battalion, Tolti Pakistan ${ }^{* * * *}$ Quaid-e-Azam International Hospital, \\ Islamabad Pakistan, ${ }^{* * * * * J i n n a h ~ P o s t g r a d u a t e ~ M e d i c a l ~ C e n t r e, ~ K a r a c h i ~ P a k i s t a n ~}$
}

\begin{abstract}
Objective: To compare the effect of age, gender and duration of diabetes mellitus on thyroid dysfunction in patients of diabetic

Study Design: Comparative cross sectional study.

Place and Duration of Study: Medicine department, Combined Military Hospital, Peshawar, from Oct 2015 to Oct 2017.

Methodology: The study was conducted after taking permission from hospital ethical review committee. Information was entered in the proforma, which included demographic information like age in years, gender, duration of disease and type of diabetes mellitus (DM) type. Already diagnosed patients with diabetes mellitus were included in the study. Thyroid function tests were carried out by standard laboratory procedure.

Results: A total of 179 patients were included in our study, $114(63.7 \%)$ were female and 65 (36.3\%) were male. Mean body mass index in our study was $29.85 \pm 3.88 \mathrm{~kg} / \mathrm{m}^{2}$. Out of 179 patients $46(25.7 \%)$ had thyroid dysfunction (TD). Out of the 46 patients with thyroid dysfunction, 14 patients $(7.8 \%)$ had clinical hypothyroidism, while subclinical hypothyroidism was seen in 25 patients (14\%). Clinical hyperthyroidism was reported in $3(1.7 \%)$, while subclinical hyperthyroidism in $4(2.2 \%)$ patients respectively.

Conclusion: Age of patients, gender and duration of disease affect thyroid functions in patients with diabetes mellitus. Thyroid profile should be carried out in diabetic patients whose blood sugar level is difficult to control.
\end{abstract}

Keywords: Diabetes mellitus, Hypoglycemia, Hypothyroidism, Thyroid dysfunction.

This is an Open Access article distributed under the terms of the Creative Commons Attribution License (https://creativecommons.org/licenses/by-nc/4.0/), which permits unrestricted use, distribution, and reproduction in any medium, provided the original work is properly cited.

\section{INTRODUCTION}

Among the endocrine disorders, worldwide, thyroid disorders and diabetes mellitus are the most frequently observed problems. Currently, the literature shows that almost $8.8 \%$ of the world population is diabetic $^{1}$. In 2016-17 national diabetic survey of Pakistan was carried out in different parts of the country and $26 \%$ of the Pakistani population was found to be diabetic 2,3 . The prevalence of thyroid dysfunctions among diabetics is not uniform globally but vary significantly in different parts of the world. In Western countries $10 \%$ of the diabetic patients have been reported to suffer from thyroid disorders ${ }^{4}$. Whereas in Asian countries the figure is much higher with $28 \%$ reported from India 5 . In Pakistan, $15 \%$ of the patients with diabetes mellitus have been reported to have associated thyroid disorder6.

A large number of patients with diabetes mellitus have associated thyroid disorders. Multiple mechanisms have been suggested for the association of thyroid disorders with diabetes mellitus, however, this association seem to be complex. With long term dia-

Correspondence: Dr Kamran Ali, Medical Specialist, Combined Military Hospital, Hyderabad Pakistan

Received: 07 Apr 2019; revised received: 20 Aug 2019; accepted: 26 Aug 2019 betes mellitus, hormonal secretions activity of pituitary and thyroid glands is altered due structural changes in the secretory mechanism ${ }^{7}$. A blunted response to different hormones is observed throughout the hypothalamus, pituitary and thyroid axis in patients with diabetes mellitus. In diabetic patients, decrease synthesis of thyrotropin releasing hormone (TRH) from hypothalamus has been observed. One theory is that the reason for hypothyroidism in these patients may be decrease secretion of TRH. There are several mechanisms by which metabolism of glucose is affected by thyroid hormones. Iodothyronines, in high concentration, blunt the response to insulin and thus prone to diabetes mellitus. Similarly, in low concentration Iodothyronines play a protective role against diabetes mellitus. In hyperthyroid states, high basal metabolic rate leads to increased rate of degradation of insulin, as a result the half-life of insulin is decreased, rendering patient susceptible to hyperglycemic state. Thus both low and high level of thyroid hormones alters diabetic pathology $y^{8,9,10}$.

The rationale of our study was to find out the effect of age, gender and duration of diabetes mellitus on thyroid dysfunction. If gender differences and duration of diabetes mellitus are found to affect thy- 
roid dysfunctions significantly, then thyroid functions test may be recommended in these patients routinely. Also treating thyroid disorders concomitantly in diabetic patients may reduce mortality and morbidity in these patients.

\section{METHODOLOGY}

This comparative cross-sectional study was carried out in the department of medicine, $\mathrm{CMH}$, Peshawar, from Oct 2015 to Oct 2017. WHO calculator was used to calculate sample size. By taking prevalence $13.4 \%, 8$ margin of error $=5 \%$ and confidence level 'CI 95\%, total sample size of 179 patients was calculated. The sampling technique used was nonprobability consecutive sampling. Patient included in the study were of either gender with age group of 20-60 years and having diabetes mellitus type 1 with duration of $>1$ and known case of diabetes mellitus type 2 with duration of more than one year. Age group was divided into 4 categories: $20-30$ years, $31-40$ years, 41-50 years and 51-60 years. Similarly, disease duration was divided into 3 categories: 1-4 years, 5-8 years and 9-12 years. Non consenting patients, known thyroidal disease, critically ill patients, patients with congestive cardiac failure, post myocardial infarction, pregnancy, chronic renal failure and chronic liver disease were excluded from the study.

This study was conducted after approval from ethics review committee. Consenting cases, meeting inclusion criteria were enrolled in the study from the Medicine Department, Combined Military Hospital Peshawar. Permission from the institutional ethical review committee was taken prior to conducting the study. Informed consent was obtained from all the patients for assigning them to the study and using their data in research. Blood sample were collected in a sterile manner after an overnight fast for serum thyroid stimulating harmon (TSH) and functional T4 (FT4) levels. Thyroid function were analyzed by RIA (Radio-immunoassay). Baseline laboratory investigations including the complete blood count, glycosylated hemoglobin $(\mathrm{HbA} 1 \mathrm{c})$ were also carried out. The findings of variables as mentioned above were entered in proforma attached as annexure.

Presence of any above condition was labeled as Thyroid dysfunction. SPSS-20 was used to analyze the data. Mean \pm SD were calculated for age, duration of disease and body mass index. Percentages and frequencies were calculated for gender and different thyroid dysfunctions. Chi square test was applied and $p$ value $\leq 0.05$ was taken significant.

\section{RESULTS}

A total of 179 patients were included in our study. Age of study cohort ranged from 24-55 years. Mean age of the patients was $41.58 \pm 9.42$ years. Of the total patients, $65(36.3 \%)$ were male and $114(63.7 \%)$ were female. Mean duration of disease (in years) of study group was $6.80 \pm 2.54$ years. Mean BMI in our study was $29.85 \mathrm{~kg} / \mathrm{m}^{2}$ with the standard deviation of \pm 3.88 . Forty six $(25.7 \%)$ patients had thyroid dysfunction and $133(74.3 \%)$ had no thyroid dysfunction. Frequency distribution of age showed that 35 (19.6\%), 42 (23.5\%), $66(36.9 \%)$ and $36(20.1 \%)$ patients were in age group 20-30 years, 31-40 years, 41-50 years and 51-60 years respectively. Duration of disease of 41 (22.9\%) patients were between 1-4 years, $94(52.5 \%)$ patients were between 5-8 years and $44(24.6 \%)$ patients were between 9-12 years. Fifty (27.9\%) patients had T1DM and 129 (72.1\%) had T2DM (table-I, II \& III).

Table-I: Thyroid dysfunction according to age $(\mathrm{n}=179)$.

\begin{tabular}{l|c|c|c}
\hline \multirow{2}{*}{ Age (Years) } & \multicolumn{2}{|c|}{ Thyroid Dysfunction } & \multirow{2}{*}{$\begin{array}{c}\boldsymbol{p} \text { - } \\
\text { value }\end{array}$} \\
\cline { 2 - 3 } & Yes & No & \\
\hline $20-30$ & $07(20 \%)$ & $28(80 \%)$ & \\
\hline $31-40$ & $08(19 \%)$ & $34(81 \%)$ & \multirow{2}{*}{0.19} \\
\hline $51-50$ & $23(34.8 \%)$ & $43(65.2 \%)$ & \\
\hline $51-60$ & $08(22.2 \%)$ & $28(77.8 \%)$ & \\
\hline Total & $46(25.7 \%)$ & $133(74.3 \%)$ & \\
\hline
\end{tabular}

Table-II: Thyroid dysfunction according to gender (n=179).

\begin{tabular}{l|c|c|c}
\hline \multirow{2}{*}{ Gender } & \multicolumn{2}{|c|}{ Thyroid Dysfunction } & \multirow{2}{*}{$\begin{array}{c}\boldsymbol{p} \text { - } \\
\text { value }\end{array}$} \\
\cline { 2 - 3 } & Yes & No & \multirow{2}{*}{0.41} \\
\hline Male & $19(29.2 \%)$ & $46(70.8 \%)$ & \\
\hline Female & $27(23.7 \%)$ & $87(76.3 \%)$ & \multirow{2}{*}{ Total } \\
\hline
\end{tabular}

Table-III: Thyroid dysfunction according to duration of disease $(n=179)$.

\begin{tabular}{l|c|c|c}
\hline \multirow{2}{*}{$\begin{array}{l}\text { Duration of } \\
\text { Disease (yrs) }\end{array}$} & \multicolumn{2}{|c|}{ Thyroid Dysfunction } & \multirow{2}{*}{$\begin{array}{c}p \text { - } \\
\text { value }\end{array}$} \\
\cline { 2 - 3 } & Yes & No & \\
\hline 01-Apr & $09(22 \%)$ & $32(88 \%)$ & \\
\hline $05-$ Aug & $27(28.7 \%)$ & $67(71.3 \%)$ & \multirow{2}{*}{0.62} \\
\hline 09-Dec & $10(22.7 \%)$ & $34(77.3 \%)$ & \\
\hline Total & $46(25.7 \%)$ & $133(74.3 \%)$ & \\
\hline
\end{tabular}

\section{DISCUSSION}

The fact that diabetes mellitus and thyroid dysfunctions are interlinked is well established. Despite such a strong association between both endocrinopathies, routine screening for thyroid dysfunction in diabetic patients is not carried out ${ }^{11,12}$. This study focused on co-existence of both endocrine disorders. In our study, we reported that 46 out of the 179 patients $(25.7 \%)$ had thyroid dysfunction in all. This reflect a 
significant prevalence of TD among our diabetic patients, both type 1 and 2, when compared to other studies. Perros et al, screened diabetic patients for thyroid dysfunctions by detecting serum levels of thyroid stimulating hormone and free thyroxin. Total number of patients included in their study were 1310 and 13.4\% patients were found to have thyroid dysfunctions. Additionally, it was shown that female with diabetes mellitus were frequently found $(31.4 \%)$ to have thyroid disorders as compared to male ${ }^{13}$. Similarly, a Greek study also looked for the prevalence of thyroid dysfunctions in diabetic patients. It found that $12.3 \%$ of diabetic patients had associated thyroid disorders. Again female with diabetes had highest prevalence of thyroid dysfunction. Another finding in their study was that dyslipidemia was more prevalent in thyroid dysfunctions patients and these patients had increase BMI ${ }^{14}$. A study conducted in Nigeria by Udiong et al, reported that among diabetic patients the prevalence of thyroid disorders was $46.5 \%$ : of which, hypothyroidism was reported in $26.6 \%$, while hyperthyroidism in $19.9 \%$ of diabetics ${ }^{15}$. None of the study has reported such high prevalence of TD in DM so far. Geffari et al ${ }^{16}$ reported a prevalence of $28.5 \%$ and a Spainish study conducted by Diez et al ${ }^{17}$, reported $25.3 \%$ of overall thyroid dysfunction in T2DM, while we reported overall thyroid dysfunction in $25.7 \%$ of diabetic patients both type 1 and 2 . The commonest thyroid disorder in this study, was sub-clinical hypothyroidism in $14.0 \%$ diabetic patients, while Perros reported $4.8 \%$, Geffari et al, reported $9.5 \%$ and Diez et al, reported $10.7 \%$ of SChypo respectively. The prevalence of clinical hypothyroidism was $7.8 \%$ in this study, while Geffari reported $15.3 \%$, Diez $15.1 \%$ and Perros $0.9 \%$ respectively.

As far as hyperthyroidism is concerned, clinical hyperthyroidism was seen in $1.7 \%$ and sub-clinical hyperthyroidism in $2.2 \%$ in our study diabetic patients, while Diez reported $3.5 \%$ and Geffari reported $0.5 \%$ of hyperthyroidism in their studies respectively. Almost all studies have shown a predominance of female gender, whenever co-existence of both disorders is seen. We also reported the predominance of female gender. Although TD was more prevalent with increasing age but statistically it was not significant, thus our study also denied the co-relation between increasing age and TD, as what has been reported by certain studies. Additionally, the association of TD with both types of diabetes showed that the prevalence of TD was seen in $6.7 \%$ of T1DM, and $18.99 \%$ of T2DM. Among patients with diabetes mellitus, thyroid autoimmunity is strongly associated with thyroid dysfunction: the co- existence of the two is explainable due to the wellknown fact that both disorders share common autoimmune etiology. The interaction between thyroid hormones and insulin is complex. In diabetic patients the production of thyrotropin releasing hormone is reduced and this may be the reason for decrease synthesis of thyroid hormone in patients with diabetes mellitus. Insulin is an anabolic hormone and increases serum level of free thyroxin that may render a patient to develop hyperthyroidism per se. Also it has been shown that some drug used for the treatment of diabetes mellitus, like phenylthioureas, increase level of thyroid stimulating hormone by suppressing serum level of free thyroxin. Serum level of thyrotropin releasing hormone and thyroid stimulating is altered by serum sugar level of diabetic patients which in turn is in affected by insulin. The presence of both hypothyroidism and hyperthyroidism in our study cohort may also be due to altered production and secretion of thyrotropin releasing hormone. Failure to recognize the TD in diabetic patients may be primary cause for poor glycemic control. One of the study had shown a significant co-relation between TSH and $\mathrm{HbA1c}$ (glycated hemoglobin), when glycemic control was evaluated in patients with SC-hypo ${ }^{18}$. Despite the fact that, we observed the TD in patients with both uncontrolled and controlled sugars, the more patients in the uncontrolled sugars group had TD, compared to those with control basal sugar levels. Further studies are needed to establish this corelation.

On the basis of result we would like to recommend that patients with diabetes mellitus should be routinely screened for thyroid disorders. Additional studies are required from our region, to establish this co-existence of the two commonest endocrine disorders, so that an effective screening, diagnostic and treatment strategy could be made.

\section{CONCLUSION}

Age of patients, gender and duration of disease affect thyroid functions in patients with diabetes mellitus. A high index of suspicion should always be kept in mind for TD in diabetic patients, especially difficult to treat diabetes.

\section{CONFLICT OF INTEREST}

This study has no conflict of interest to be declared by any author.

\section{REFERENCES}

1. Ogurtsova K, da Rocha Fernandes JD, Huang Y, Linnenkamp U, Guariguata L, Cho NH, et al. IDF Diabetes Atlas: Global esti- 
mates for the prevalence of diabetes for 2015 and 2040. Diabetes Res Clin Pract 2017; 128(1): 40-50.

2. Basit A, Fawwad A. Prevalence of diabetes, pre-diabetes and associated risk factors: second National Diabetes Survey of Pakistan (NDSP), 2016-2017. BMJ open 2018; 8(1): e020961-65.

3. Basit A, Fawwad A, Baqa K. Pakistan and diabetes-A country on the edge. Diabetes Res Clin Pract 2019; 147(1): 166-68.

4. Nederstigt C, Corssmit EP, de Koning EJ, Dekkers OM. Incidence and prevalence of thyroid dysfunction in type 1 diabetes. J Diabetes Complica 2016; 30(2): 420-25.

5. Ozair M, Noor S, Raghav A, Siddiqi SS, Chugtai AM, Ahmad J. Prevalence of thyroid disorders in North Indian type 2 diabetic subjects: A cross sectional study. Diabetes Metab Syndr 2018; 12(1): 301-04.

6. Sajid N, Riaz M, Fawwad A, Basit A. Thyroid dysfunction in subjects with type 1 diabetes at a tertiary care unit of Karachi, Pakistan. clinical epidemiology and global health 2019. [Internet]. Available at: https:// doi.org/10.1016/j.cegh.2019.01.001.

7. De Vito P, Candelotti E, G Ahmed R, Luly P, J Davis P, Incerpi S, et al. Role of thyroid hormones in insulin resistance and diabetes. Immunology, endocrine \& metabolic agents in medicinal chemistry (formerly current medicinal chemistry-immunology, Endocrine and Metabolic Agents). 2015; 15(1): 86-93.

8. Shpakov AO. Molecular mechanisms of the relationship between thyroid dysfunctions and diabetes mellitus. J Evolutionary Biochemistry Physiol 2018; 54(4): 257-66.

9. Uppal V, Vij C, Bedi GK, Vij A, Banerjee BD. Thyroid disorders in patients of type 2 diabetes mellitus. Ind J Clinical Biochem
2013; 28(4): 336-41.

10. Ghigo E, Porta M. Diabetes secondary to endocrine and pancreatic disorders. front diabetes. Basel Karger, 2014; 22(1): pp 52-63.

11. Biondi B, Kahaly GJ, Robertson RP. Thyroid dysfunction and diabetes mellitus: two closely associated disorders. Endocrine reviews 2019; 40(3): 789-24.

12. Jali MV, Kambar S, Jali SM, Pawar N, Nalawade P. Prevalence of thyroid dysfunction among type 2 diabetes mellitus patients. diabetes \& metabolic syndrome: Clinical Research \& Reviews. 2017; 11(1): S105-8

13. Perros P, McCrimmon RJ, Shaw G. Frequency of thyroid dysfunction in diabetic patients: value of annual screening. Diabet Med. 1995; 12(7): 622-27.

14. Papazafiropoulou A, Sotiropoulos A, Kokolaki A. Prevalence of thyroid dysfunction among Greek Type 2 diabetic patients attending an outpatient clinic. J Clin Med Res 2010; 2(2): 75-78.

15. Udiong $\mathrm{CE}$, Udoh $\mathrm{AE}$, Etukudoh ME. Evaluation of thyroid function in diabetes mellitus in Calabar, Nigeria. Indian J Clin Biochem. 2007; 22(2): 74-78.

16. Al-Geffari M, Ahmad NA, Al-Sharqawi AH, Youssef AM, AlNaqeb D, Al-Rubeaan K. Risk factors for thyroid dysfunction among type 2 diabetic patients in a highly diabetes mellitus prevalent society. Inter J Endocrinol 2013; 2013.

17. D'iez JJ, Iglesias P. Prevalence of thyroid dysfunction in patients with type 2 diabetes. Experiment Clin Endocrinol Diab 2011; 119(4): 201-7.

18. Beciragic A, Bilic-Komarica E. Impotance of HbA1c control in patients with hypothyroidism. Mat Soc Med 2012; 24(4): 212-15. 